\title{
CT-Morphologie der COVID-19-Pneumonie
}

Jan Schaible, Stefanie Meiler, Florian Poschenrieder, Benedikt Pregler,

Gregor Scharf, Okka W. Hamer

\section{Einleitung}

Die durch SARS-CoV-2 verursachte Coronavirus Disease 2019 (COVID-19) hat inzwischen den Status einer Pandemie erreicht. Der definitive Nachweis erfolgt über die RT-PCR aus Proben der oberen oder unteren Atemwege. Die Morphologie der COVID-19-Pneumonie in der CT ist bemerkenswerterweise bei vielen Patienten ähnlich, sodass sie die Diagnosestellung unterstützen kann. Zudem ermöglicht die CT eine Bestimmung des Ausmaßes der Parenchymveränderungen, eine Verlaufsbeurteilung und die Erkennung evtl. Komplikationen. In diesem Bildessay wird die CT-Morphologie der COVID-19-Pneumonie beschrieben.

Gemäß der Statements der nationalen und internationalen thoraxradiologischen Gesellschaften ist die bildgebende Methode der Wahl bei Patienten mit Verdacht auf oder gesicherter COVID-19-Pneumonie die CT des Thorax [1] (https://www.drg.de/de-DE/5995/covid-19/). Die Thoraxübersichtsaufnahme ist initial nicht empfohlen, da ihre Sensitivität zu gering ist [2]. Sie kann jedoch bei Verlaufskontrollen zum Einsatz kommen. Die CT sollte

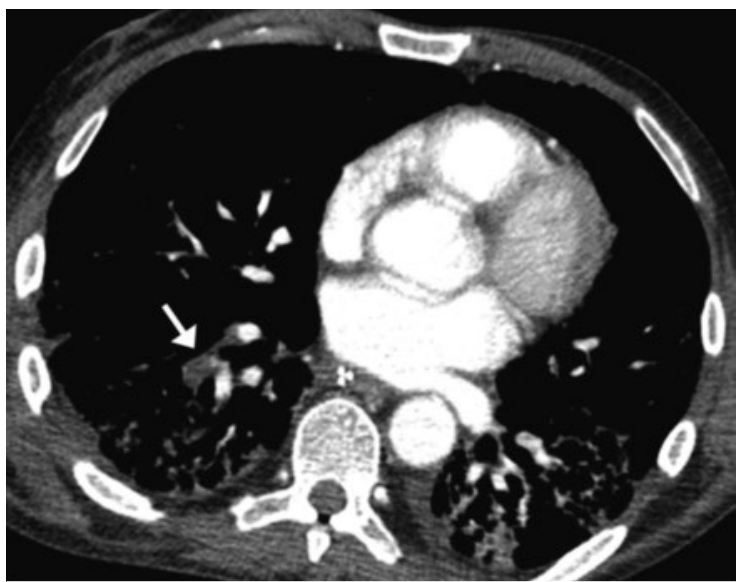

- Abb. 1 COVID-19-Pneumonie kompliziert durch Lungenembolie. Kontrastmittelgestützte dünnschichtige Volumen-CT. MPR in axialer Ebene. In einer Subsegmentarterie des rechten Unterlappens zeigt sich eine Kontrastmittelaussparung (Pfeil) im Sinne einer Lungenembolie. Der Patient litt an einer fortgeschrittenen COVID-19Pneumonie. als native dünnschichtige Volumen-CT in Niedrigdosistechnik durchgeführt werden [3]. Allerdings mehrt sich die Evidenz, dass eine Infektion mit SARS-CoV-2 zu einer Hyperkoagulopathie mit vermehrtem Auftreten von thrombotischen und embolischen Ereignissen führt ( $\triangleright$ Abb. 1) [4-6]. Bei steigendem Wert der D-Dimere oder anderweitigen Verdachtsmomenten auf eine Lungenembolie sollte daher Kontrastmittel verabreicht werden.

\section{CT-Morphologie der COVID-19-Pneumonie}

Die Morphologie der COVID-19-Pneumonie in der CT ist bemerkenswerterweise bei vielen Patienten ähnlich. Meist liegen Milchglastrübungen oder ein Mischbild aus Milchglastrübungen und Konsolidierungen vor ( $\triangleright$ Abb. 2, Abb. 3, Abb. 4, Abb. 5, Abb. 6, Abb. 7) [7-15]. Die Milchglastrübungen können von einem feinen Netzmuster (Retikulationen) überlagert sein, was als Crazy Paving bezeichnet wird ( $\bullet$ Abb. 4, Abb. 8). Vielfach ist beschrieben, dass die Gefäße innerhalb der Verdichtungen oder

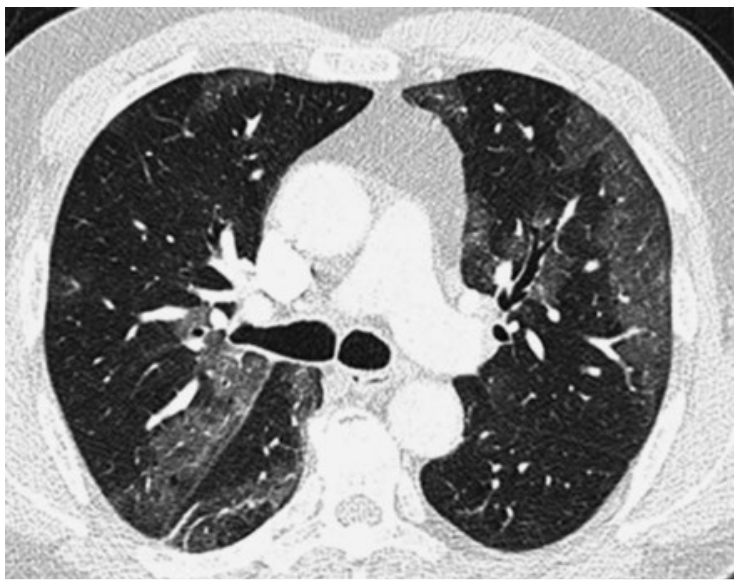

- Abb. 2 COVID-19-Pneumonie. Kontrastmittelgestützte dünnschichtige Volumen-CT. MPR in axialer Ebene. In der Peripherie beider Mittelfelder zeigen sich multifokale Milchglastrübungen. Sie sind geografisch konfiguriert. Positives Aerobronchogramm. 


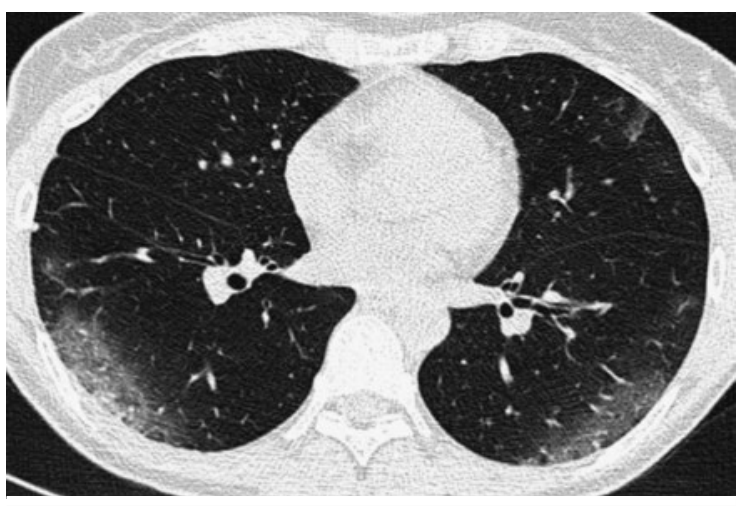

- Abb. 3 COVID-19-Pneumonie. Native dünnschichtige Volumen-CT. MPR in axialer Ebene. In der Peripherie beider Mittelfelder zeigt sich ein Mischbild aus Milchglastrübungen und Konsolidierungen. Die posterioren Segmente sind dominant betroffen.

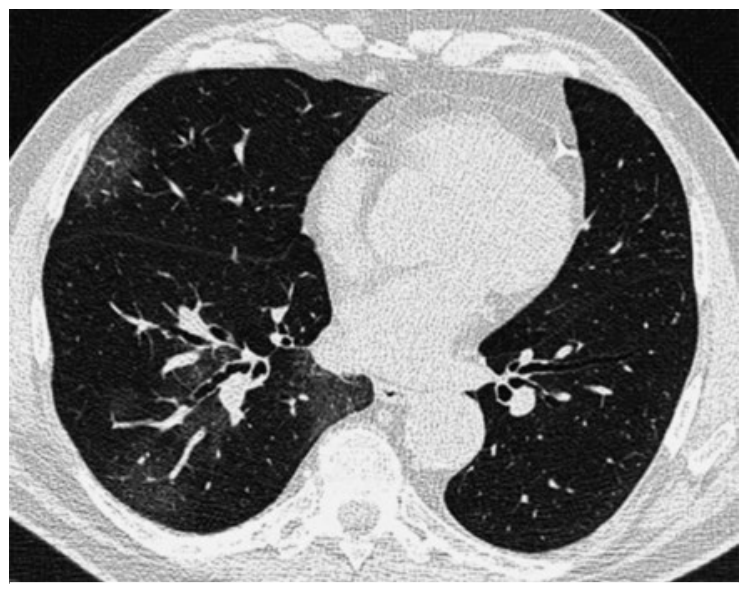

- Abb. 4 COVID-19-Pneumonie. Native dünnschichtige Volumen-CT. MPR in axialer Ebene. Multifokale Milchglastrübungen im rechten Unterfeld (die linke Lunge war ebenso betroffen, nicht gezeigt). Die Milchglastrübungen sind zum Teil von Retikulationen überlagert, was als Crazy Paving bezeichnet wird. Sie sind rund konfiguriert und liegen bevorzugt in der Peripherie der Lunge.

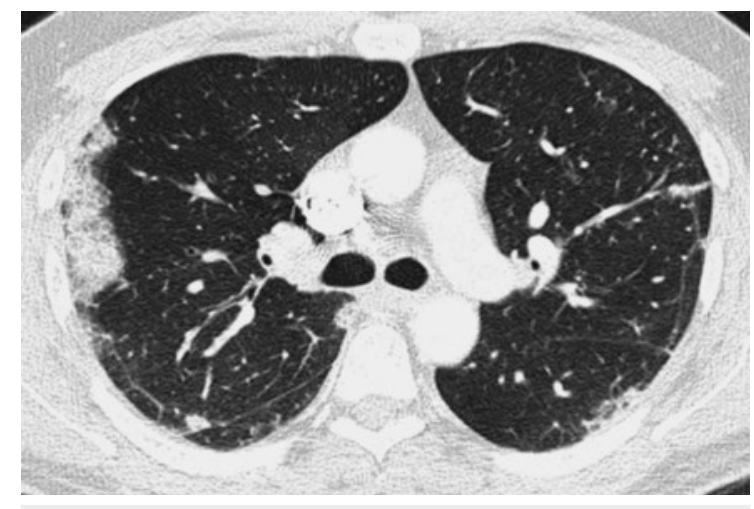

- Abb. 5 COVID-19-Pneumonie. Kontrastmittelgestützte dünnschichtige Volumen-CT. MPR in axialer Ebene. Fleckförmige bis konfluierende sowie streifige Konsolidierungen in der Peripherie beider Mittelfelder. Die Verdichtungen sind teils geografisch konfiguriert.

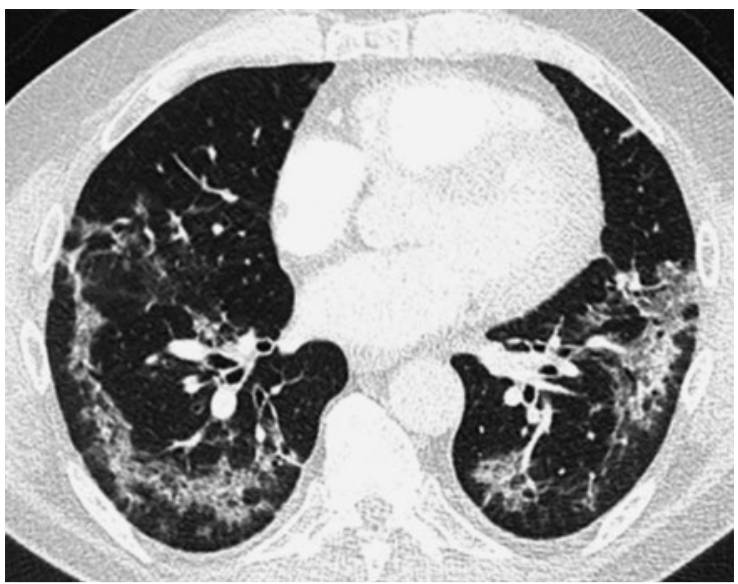

- Abb. 6 COVID-19-Pneumonie. Kontrastmittelgestützte dünnschichtige Volumen-CT. MPR in axialer Ebene. In der Peripherie beider Mittelfelder zeigt sich ein Mischbild aus Milchglastrübungen und Konsolidierungen. Die Verdichtungen sind zum Teil rund, zum Teil geografisch konfiguriert. Positives Aerobronchogramm. Der unmittelbare subpleurale Raum ist ausgespart, was eher in späteren Stadien der Fall ist. Die posterioren Segmente sind dominant betroffen.

schadens (diffuse alveolar damage, DAD) kommen ( Abb. 10).

Liegt die beschriebene CT-Morphologie vor, kann in Zeiten einer hohen Umgebungsprävalenz (Pandemie) und passender klinischer Symptomatik mit hoher Wahrscheinlichkeit von einer COVID-19-Pneumonie ausgegangen werden. Dies ist hilfreich, da die Sensitivität der CT höher sein kann als die des Goldstandards der Reversen Transkriptase-Polymerasekettenreaktion (RT-PCR) [16]. 


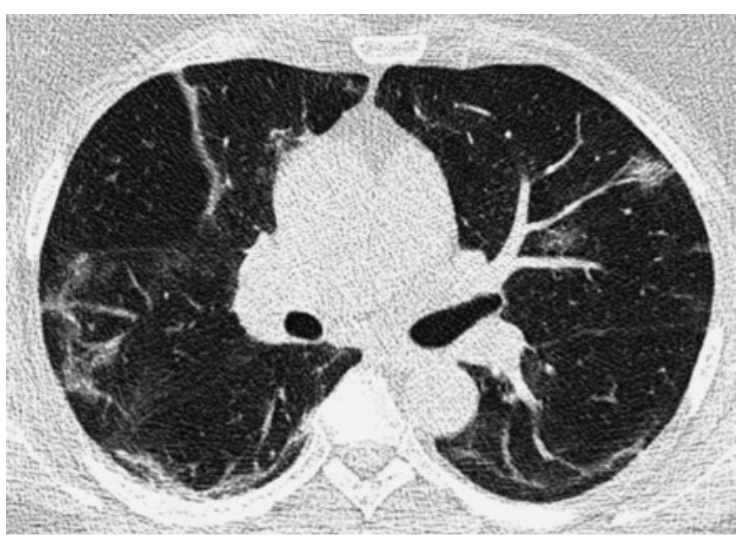

Abb. 7 COVID-19-Pneumonie. Native dünnschichtige Volumen-CT in Low-Dose-Technik. MPR in axialer Ebene. Mischbild aus fleckförmigem Milchglas und streifigen Konsolidierungen. Die Verdichtungen liegen überwiegend in der Peripherie der Lunge, vor allem posterior. Der unmittelbare subpleurale Raum ist ausgespart, was eher in späteren Stadien der Fall ist.

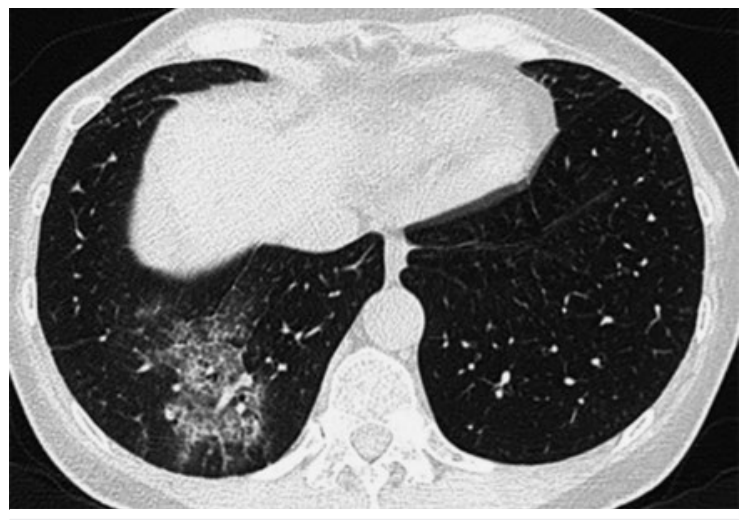

- Abb. 8 COVID-19-Pneumonie. Native dünnschichtige Volumen-CT. MPR in axialer Ebene. Im rechten Unterlappen kommt eine annähernd rund konfigurierte Milchglastrübung mit überlagernden Retikulationen im Sinne eines Crazy Paving zur Darstellung. Positives Aerobronchogramm. Das dominante Gefäß innerhalb der Verdichtung ist dilatiert.

In einer jüngst veröffentlichten Studie aus Italien war die RT-PCR bei 96 von 158 Patienten initial negativ, die CT bei 42 dieser 96 Patienten positiv [17]. Andererseits schließt eine unauffällige CT COVID-19 nicht aus.

Zu bedenken ist, dass nicht alle Patienten die oben beschriebene suggestive Morphologie zeigen. Es kann auch das unspezifische Bild einer atypischen Pneumonie vor-

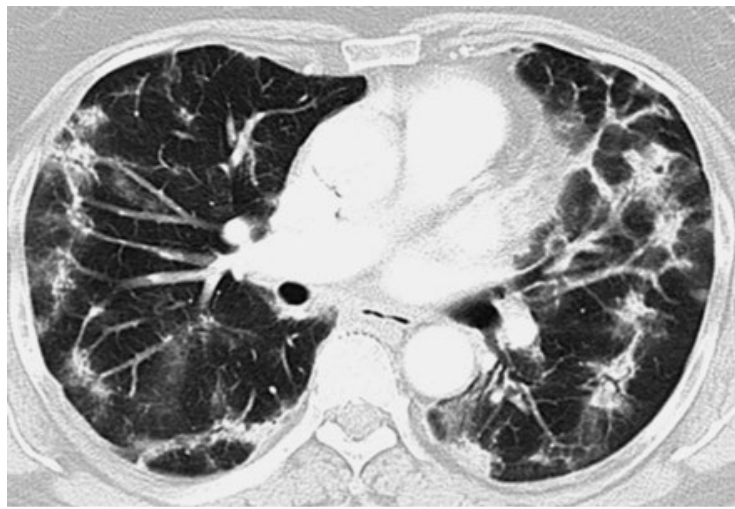

- Abb. 9 Differenzialdiagnose kryptogen organisierende Pneumonie. Kontrastmittelgestützte Volumen-CT. MPR in axialer Ebene. In der Peripherie beider Mittelfelder zeigt sich ein Mischbild aus Konsolidierungen und Milchglastrübungen. Die Verdichtungen sind zum Teil fleckig, zum Teil streifig konfiguriert und liegen in der Peripherie der Lunge. Positives Aerobronchogramm.

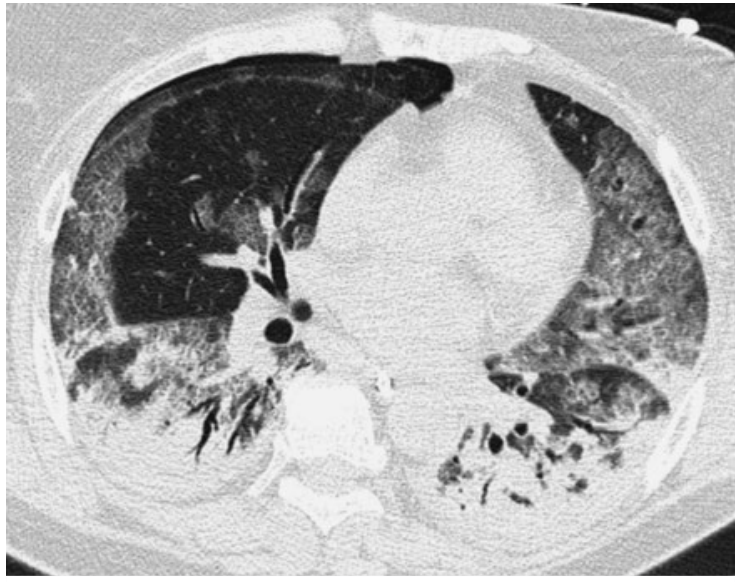

- Abb. 10 Fortgeschrittene COVID-19-Pneumonie. Native dünnschichtige Volumen-CT. MPR in axialer Ebene. In beiden Lungen zeigen sich dorsal betonte Konsolidierungen und ventral davon gelegenes Crazy Paving. Positives Aerobronchogramm. Das Bild passt zu einem diffusen Alveolarschaden. Nebenbefundlich schmaler Pneumothorax rechts.

liegen, das keinen Rückschluss auf den Erreger erlaubt ( $\bullet$ Abb. 11, Abb. 12). Wertvoll zu wissen ist, welche Zeichen bisher bei der COVID-19-Pneumonie nicht gesehen wurden. Hierzu gehören Noduli, Tree-in-Bud, Kavernen, Bronchialwandverdickungen und Mucus Plugging. Pleuraergüsse oder eine mediastinale Lymphadenopathie wurden nur bei unter $10 \%$ der Patienten beobachtet. 


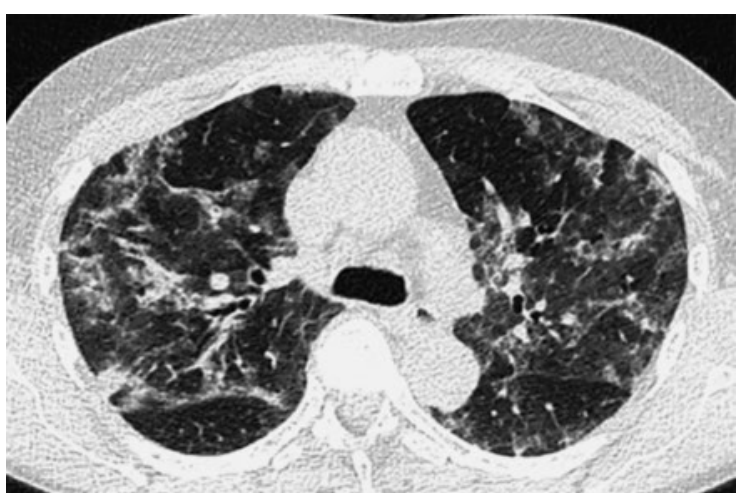

- Abb. 11 COVID-19-Pneumonie. Native dünnschichtige Volumen-CT. MPR in axialer Ebene. In beiden Mittelfeldern kommt ein Mischbild aus zum Teil fleckigen, zum Teil konfluierenden Milchglastrübungen und Konsolidierungen zur Darstellung. Positives Aerobronchogramm. In axialer und koronarer (nicht gezeigt) Ebene sind beide Lungen diffus betroffen. Das Bild passt zu einer atypischen Pneumonie, wobei keine Zuordnung zu einem Erreger möglich ist.

\section{Zusammenfassung}

Der Goldstandard für COVID-19-Pneumonie ist die RT-PCR. Allerdings ist die CT bei einigen Fällen sensitiver als der Goldstandard. Dennoch sollte die CT nicht als Screening-Methode eingesetzt werden. Die CT ist jedoch sehr hilfreich, um die Patientenströme zu lenken. Gemäß der CT-Morphologie scheint es nämlich möglich, Patienten mit hoher Prätestwahrscheinlichkeit während der Pandemie in 3 Gruppen einzuteilen:

1. CT-Morphologie suggestiv für eine COVID-19-Pneumonie

2. CT-Morphologie spricht für eine atypische Pneumonie, keine Zuordnung zu einem Erreger möglich

3. CT-Morphologie spricht gegen eine COVID-19-Pneumonie $[18,19,20]$.

Die CT kann zudem das Ausmaß der Lungenparenchymbeteiligung erfassen und evtl. Komplikationen wie eine Superinfektion oder Lungenembolie erkennen.

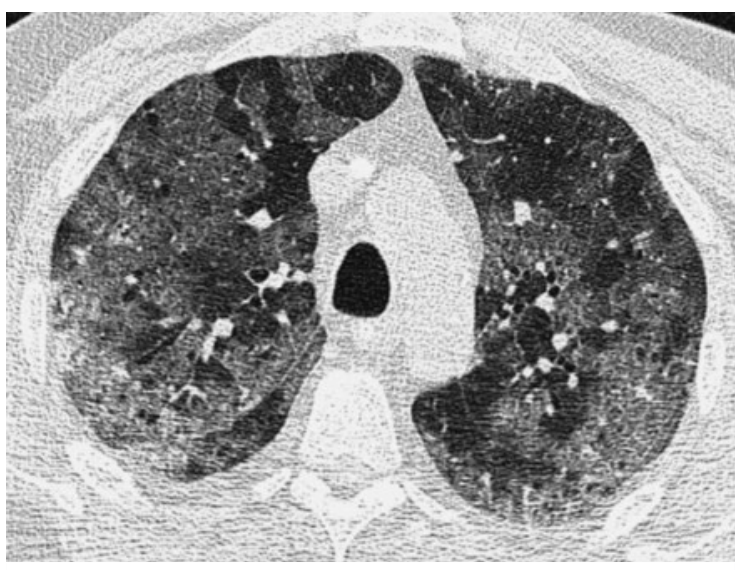

- Abb. 12 COVID-19-Pneumonie. Native dünnschichtige Volumen-CT in Low-Dose-Technik. MPR in axialer Ebene. Ausgedehnte, zum Teil geografisch konfigurierte Milchglastrübungen und Crazy Paving. Diskret ausgebildete fleckige Konsolidierungen. Positives Aerobronchogramm. In axialer und koronarer (nicht gezeigt) Ebene sind beide Lungen diffus betroffen. Das Bild passt zu einer schweren atypischen Pneumonie, wobei keine Zuordnung zu einem Erreger möglich ist.

Interessenkonflikt

Die Autorinnen/Autoren geben an, dass keine Interessenkonflikt besteht.

Korrespondenzadresse

Prof. Dr. med. Okka W. Hamer

Universitätsklinikum Regensburg

Institut für Radiologie

Franz-Josef-Strauß-Allee 11

93046 Regensburg

okka.hamer@klinik.uni-regensburg.de

und

Lungenfachklinik Donaustauf

Abteilung für Radiologie

Ludwigstr. 68

93093 Donaustauf 
[1] Rubin GD, Haramati LB, Kanne JP et al. The Role of Chest Imaging in Patient Management during the COVID-19 Pandemic: A Multinational Consensus Statement from the Fleischner Society. Radiology 2020. doi:10.1148/radiol.2020201365

[2] Choi H, Qi X, Yoon SH et al. Extension of Coronavirus Disease 2019 (COVID-19) on Chest CT and Implications for Chest Radiograph Interpretation. Radiology: Cardiothoracic Imaging 2020; 2: e200107. doi:10.1148/ryct.2020200107

[3] Antoch G, Urbach H, Mentzel H] et al. SARS-CoV-2/COVID-19: Empfehlungen für die Radiologische Versorgung - Eine Stellungnahme, der Deutschen Röntgengesellschaft (DRG), der Deutschen Gesellschaft für Neuroradiologie (DGNR), der Gesellschaft für Pädiatrische Radiologie (GPR), der Deutschen Gesellschaft für Interventionelle Radiologie (DeGIR), des Berufsverbands der Neuroradiologen (BDNR), und des Berufsverbands der Radiologen (BDR). Fortschr Röntgenstr 2020; 192: 418-421. doi:10.1055/a-1149-3625

[4] Cui S, Chen S, Li X et al. Prevalence of venous thromboembolism in patients with severe novel coronavirus pneumonia. J Thromb Haemost 2020. doi:10.1111/jth.14830

[5] Klok FA, Kruip MJHA, van der Meer NJM et al. Incidence of thrombotic complications in critically ill ICU patients with COVID-19. Thromb Res 2020. doi:10.1016/j.thromres.2020. 04.013

[6] Xie Y, Wang X, Yang P et al. COVID-19 Complicated by Acute Pulmonary Embolism. Radiology: Cardiothoracic Imaging 2020; 2: e200067. doi:10.1148/ryct.2020200067

[7] Bernheim A, Mei X, Huang M et al. Chest CT Findings in Coronavirus Disease-19 (COVID-19): Relationship to Duration of Infection. Radiology 2020. doi:10.1148/radiol.2020200463

[8] Chung,M, Bernheim A, Mei X et al. CT Imaging Features of 2019 Novel Coronavirus (2019-nCoV). Radiology 2020; 295: 202-207. doi:10.1148/radiol.2020200230

[9] Han R, Huang L, Jiang $H$ et al. Early Clinical and CT Manifestations of Coronavirus Disease 2019 (COVID-19) Pneumonia. AJR Am J Roentgenol 2020. doi:10.2214/AJR.20.22961

[10] Kong W, Agarwal PP. Chest Imaging Appearance of COVID-19 Infection. Radiology: Cardiothoracic Imaging 2020; 2: e200028. doi:10.1148/ryct.2020200028
[11] Lei P, Fan B, Yuan Y. The evolution of CT characteristics in the patients with COVID-19 pneumonia. J Infect 2020. doi:10.1016/j.jinf.2020.03.014

[12] Li K, Wu J, Wu F et al. The Clinical and Chest CT Features Associated with Severe and Critical COVID-19 Pneumonia. Invest Radiol 2020. doi:10.1097/RLI.0000000000000672

[13] Li Y, Xia L. Coronavirus Disease 2019 (COVID-19): Role of Chest CT in Diagnosis and Management. AJR Am J Roentgenol 2020. doi:10.2214/AJR.20.22954

[14] Ng MY, Lee EYP, Yang J et al. Imaging Profile of the COVID-19 Infection: Radiologic Findings and Literature Review. Radiology: Cardiothoracic Imaging 2020; 2: e200034. doi:10.1148/ ryct.2020200034

[15] Shi $\mathrm{H}$, Han X, Jiang $\mathrm{N}$ et al. Radiological findings from 81 patients with COVID-19 pneumonia in Wuhan, China: a descriptive study. Lancet Infect Dis 2020; 20: 425-434. doi:10.1016/ S1473-3099(20)30086-4

[16] Fang Y, Zhang H, Xie J et al. Sensitivity of Chest CT for COVID19: Comparison to RT-PCR. Radiology 2020. doi:10.1148/radiol.2020200432

[17] Caruso D, Zerunian M, Polici M et al. Chest CT Features of COVID-19 in Rome, Italy. Radiology 2020. doi:10.1148/radiol.2020201237

[18] Kanne JP, Little BP, Chung JH et al. Essentials for Radiologists on COVID-19: An Update-Radiology Scientific Expert Panel. Radiology 2020. doi:10.1148/radiol.2020200527

[19] Vogel-Claussen J, Ley-Zaporozhan J, Agarwal P et al. Recommendations of the thoracic imaging section of the German Radiological Society for clinical application of chest imaging and structured CT reporting in the COVID-19 pandemic. Fortschr Röntgenstr 2020; 192. doi:10.1055/a-1174-8378

[20] Schaible J, Meiler S, Poschenrieder F et al. Radiology of COVID19 pneumonia - pictorial essay and structured reporting. Rofo 2020; 192: 513-517. doi:10.1055/a-1164-7001

Bibliografie

DOI https://doi.org/10.1055/a-0024-5493

Radiologie up2date 2020; 20: 111-115

(c) Georg Thieme Verlag KG Stuttgart · New York ISSN 1616-0681 